\title{
Modèles nationaux d'intégration de migrant.e.s et résultats PISA: éléments pour un débat
}

Thibaut Lauwerier et Abdeljalil Akkari, Université de Genève

Il existe un débat ancien entre les tenant.e.s d'une supériorité conceptuelle de l'éducation interculturelle par rapport à l'éducation multiculturelle, et celles et ceux qui considèrent qu'il n'existe pas de différences notables entre ces deux modèles de gestion de la diversité culturelle. Dans cet article, nous analysons dans un premier temps les arguments mobilisés dans le cadre de ce débat. Dans un deuxième temps, nous utiliserons les données de l'enquête PISA pour comparer les résultats des pays ayant adopté l'interculturalisme (France, Espagne, Suisse) avec des pays utilisant le multiculturalisme (Canada, États-Unis, Royaume-Uni). La comparaison portera sur les résultats des jeunes issus de la diversité (élèves immigrés de la première et de la deuxième génération). Cette comparaison permettra d'explorer les liens éventuels entre modèles d'intégration (interculturalisme ou multiculturalisme) et performances scolaires pour voir si un modèle fait effectivement la différence sur ces questions.

\section{Introduction}

Cet article prend comme point de départ la distinction théorique entre deux modèles d'intégration, l'éducation interculturelle et l'éducation multiculturelle. Il existe un débat ancien entre les auteur.e.s qui distinguent ces deux approches et les auteur.e.s qui considèrent qu'il n' existe pas de différences notables entre ces deux modèles pour réduire l'écart de réussite scolaire entre les groupes ethnoculturels et offrir des opportunités éducatives équivalentes à tous les apprenant.e.s. Notre article vise donc à voir dans quelle mesure une distinction entre les deux modèles peut être observée en se basant sur les performances scolaires des élèves dans des pays qui sont identifiés à un de ces modèles.

La classification des pays en deux groupes, interculturel et multiculturel, est justifiée par la terminologie utilisée dans les documents officiels et professionnels nationaux. Le Canada anglophone utilise le terme «éducation multiculturelle», en particulier dans la loi sur le maintien et la valorisation du multicultura- 
lisme au Canada (1988). Aux États-Unis, le multiculturalisme est présent aussi bien dans les documents gouvernementaux que dans les textes concernant les enseignant.e.s (Stepanovich, 2017). En Espagne, en France, en Belgique et en Suisse, les documents officiels se réferent généralement aux directives du Conseil de l'Europe en matière d'interculturalisme, et la recherche utilise en principe le modèle interculturel (Catarci \& Fiorucci, 2016; Dasen \& Perregaux, 2000; Ferrer \& Akkari, 2000; Leman, 1993). Deux arguments additionnels soutiennent notre classification. D’une part, l'accès à la citoyenneté pour les migrant.e.s légaux est facilité dans le modèle multiculturaliste avec une durée de résidence exigée relativement courte (environ 3 ans) alors que l'accès à la citoyenneté dans les pays relevant du modèle interculturaliste est plus long (5-10 ans). D'autre part, les pays du modèle multiculturaliste se considèrent et s'assument comme des pays d'immigration alors que les pays du modèle interculturaliste reçoivent de nombreux migrant.e.s sans considérer être un pays d'immigration. Notre classification utilise essentiellement le niveau macro des politiques éducatives, et fait le pari avancé par Taylor (2012) que les sociétés utilisant le multiculturalisme se voient et se projettent différemment que celles qui adoptent l'interculturalisme. Néanmoins, nous reconnaissons l'existence d'autres niveaux d'analyse des politiques de la diversité (province, ville, école etc.) pas forcément cohérents avec le niveau macro. L'exemple du Québec illustre bien la complexité d'une prise en compte de tous les niveaux de gestion de la diversité et les contradictions existantes en passant du niveau fédéral à un niveau provincial (Segura, 2011).

Ainsi, dans cet article, nous analysons, dans un premier temps, les arguments mobilisés dans le cadre du débat interculturel/multiculturel. Ensuite, nous mobilisons la littérature qui traite des liens entre performances scolaires et origines ethnoculturelles selon l'enquête PISA. Dans un troisième temps, utilisant les données de PISA 2015, nous comparons les résultats des pays ayant adopté l'interculturalisme (France, Espagne, Suisse) avec des pays utilisant le multiculturalisme (Canada, États-Unis, Royaume-Uni). La comparaison portera sur les résultats des jeunes issus.e.s de la diversité (élèves immigré.e.s des première et deuxième générations). Cela nous amènera à conclure que le lien entre modèle d'intégration et performances scolaires n'est pas évident à établir. Même s'il ressort des analyses que les élèves immigré.e.s des pays multiculturels ont sensiblement de meilleures performances scolaires, il est difficile de généraliser cette tendance de la même manière à l'ensemble de ces pays, des facteurs autres que les modèles d'intégration expliquant aussi les performances 


\section{Les fondements de l'éducation multiculturelle et de l'éducation interculturelle: divergences ou convergences?}

L'éducation multiculturelle ou interculturelle s'inscrit dans le cadre de politiques publiques plus vastes visant à mieux gérer et valoriser la diversité. Il est donc important de s'attarder sur les fondements politiques et philosophiques de ces deux orientations. D’une manière générale, nous pouvons observer que le terme "éducation multiculturelle» s'est imposé dans les pays anglo-saxons en particulier aux États-Unis, au Canada anglophone et au Royaume-Uni. En revanche, dans les pays francophones et hispanophones d'Europe, le terme consacré est «éducation interculturelle».

Le concept d'éducation multiculturelle est apparu avant l'émergence de l'éducation interculturelle. Aux États-Unis, Takaki (2012) montre que l'histoire du multiculturalisme dans le pays est une question de lutte ancienne pour l'égalité et pour la flexibilisation des lignes de démarcation entre races, ethnies et couleurs. Banks (1993) situe le développement de l'éducation multiculturelle dans le cadre du mouvement des droits civiques des années 1960-70 et distingue quatre phases de développement. La première phase est apparue avec l'introduction des études ethniques (histoire et culture des minorités ethniques) à l'école et dans les programmes de formation des enseignant.e.s. Une deuxième phase (éducation multiethnique) a émergé lorsque les enseignant.e.s intéressé.e.s par les études ethniques ont constaté que leur insertion dans les programmes scolaires et de formation des enseignant.e.s était nécessaire mais pas suffisante pour amener une réforme scolaire qui réponde aux besoins des élèves appartenant à des minorités ethniques. Le but de cette deuxième phase était d'engendrer des changements structurels et systémiques dans l'ensemble du système éducatif pour accroître l'égalité d'opportunité en matière d'éducation. Une troisième phase a eu lieu lorsque d'autres groupes victimes d'inégalités dans la société et à l'école, telles que les femmes et les personnes en situation de handicap, ont exigé l'intégration de leurs histoires, leurs cultures et leurs voix dans les programmes et la structure du système éducatif. La quatrième phase de l'éducation multiculturelle consiste en l'élaboration de théories, recherches et pratiques articulant les variables d'origine culturelle, classe et genre pour accroitre l'équité et l'égalité.

Au Canada anglophone, l'éducation multiculturelle est liée à la politique fédérale du multiculturalisme mise en place au début des années 1970 par le premier ministre Pierre-Elliott Trudeau (Day, 2000). L'idée était de reconfigurer l'identité nationale en intégrant sur un pied d'égalité l'apport de tous les groupes présents sur le sol canadien. Il s'agissait aussi de rendre justice aux peuples premiers ayant souffert d'une discrimination systématique. L'éducation multiculturelle s'est traduite par de nombreux programmes de lutte contre les discriminations, de valorisation des langues des apprenant.e.s et d'un encouragement de toutes les communautés à s'impliquer dans la gestion du système 
éducatif (Guo \& Wong, 2015). Le Royaume-Uni s’inscrit également dans le cadre du paradigme multiculturel qui peut être considéré dans ce pays comme une politique de l'État (Mathieu, 2018), bien que controversée (Ashcroft \& Bevir, 2018).

Dans deux principaux lieux d'émergence de l'éducation multiculturelle (États-Unis et Canada anglophone), cette approche met en évidence la nécessité de reconnaître l'apport de tous les groupes ethniques et culturels à l'identité nationale, et de reconnaitre les injustices que certains groupes ont subies. Pour l'école, il s'agit de promouvoir des politiques et des programmes permettant de réduire l'écart de réussite scolaire entre groupes ethnoculturels et d'offrir des opportunités éducatives équivalentes à toutes et tous les apprenant.e.s. En raison de la malléabilité de l'éducation multiculturelle, on observe une multitude d'approches qui peuvent schématiquement se situer entre une valorisation «folklorique» de la diversité et une volonté de mettre en œuvre une pédagogie critique susceptible de changer radicalement les fondements de la forme scolaire (Kymlicka, 2019).

En Europe francophone et hispanophone (France, Suisse, Belgique, Espagne), l'émergence de l'éducation interculturelle se situe plutôt dans le contexte de l'intensification des mouvements migratoires des années 1970-80. Lécole et les sociétés européennes ont été confrontées à l'arrivée massive de jeunes migrant.e.s ne maîtrisant pas la langue d'instruction des pays d'accueil. C'est le Conseil de l'Europe qui a initié la réflexion sur l'éducation interculturelle en partant $\mathrm{du}$ postulat que l'intégration sociale et scolaire est un processus à double sens. Il concerne les migrant.e.s accueilli.e.s, mais aussi les autochtones qui doivent opérer des ajustements afin de faciliter la dynamique du vivre ensemble (Porcher, 1981). L'éducation interculturelle se fonde sur l'importance des interactions et d'une relation symétrique et égalitaire entre les différents groupes. Le paradigme interculturel affiche une volonté de valoriser les cultures, mais surtout de les décloisonner et de favoriser les interactions entre les groupes (Perotti, 1994). Dans différents pays d'Europe, les ministères de l'éducation se sont saisis du paradigme interculturel pour créer des structures d'accueil et des programmes favorisant l'intégration scolaire des élèves d'origine étrangère. Au niveau de la langue par exemple, des efforts ont été consentis pour mieux enseigner les langues d'instruction aux élèves allophones, mais dans le même temps, les langues d'origine des élèves migrant.e.s ont été promues à travers l'enseignement de la langue et culture d'origine (Auger, 2007).

Il existe un débat ancien entre les tenant.e.s d'une supériorité conceptuelle de l'éducation interculturelle par rapport à l'éducation multiculturelle et celles et ceux qui considèrent qu'il n'existe pas de différences notables entre les deux modèles de gestion de la diversité culturelle et d'intégration de migrant.e.s, et leurs implications possibles en termes de réussite scolaire. De ce point de vue, les postures different entre auteur.e.s anglophones et francophones. Pour les premières/premiers, il n'existe pas vraiment de différences entre l'éducation 
multiculturelle et interculturelle. Les deux approches proposent les mêmes concepts et les mêmes objectifs (Meer \& Modood, 2012; Sleeter, 2018). Elles/ ils rejettent l'idée que l'éducation multiculturelle s'arrête au niveau de la valorisation des cultures et favoriserait la séparation entre les communautés culturelles. Par ailleurs, les auteur.e.s anglophones estiment que le multiculturalisme précède et est une condition préalable à l'interculturalisme. Enfin, elles/ils observent que le courant de la pédagogie critique ou du multiculturalisme critique et ses auteur.e.s de référence tels que Freire ou McLaren (1997) s'inscrivent dans une visée de transformation radicale de l'école et de lutte contre les inégalités. En revanche, pour les auteur.e.s francophones, éducation multiculturelle et éducation interculturelle ne peuvent être confondues (Abdallah-Pretceille, 2011; Grima Camilleri, 2002). La première s'arrêterait au niveau de la valorisation des cultures considérées comme séparées et hermétiques; engendrant même un risque de séparatisme et communautarisme. L'éducation interculturelle serait ainsi conceptuellement et pédagogiquement supérieure mettant l'accent sur les relations et les interactions interculturelles. Brahm Levey (2012) se positionne à mi-chemin: il partage l'essentiel de la thèse de Meer et Modood (2012) tout en relevant que les notions de dialogue et d'interaction sont plus présents dans le modèle interculturaliste.

Notons que l'éducation multiculturelle et l'éducation interculturelle n'abordent pas la question de la diversité religieuse de la même manière. Pour l'éducation multiculturelle, l'intégration des croyances et des convictions religieuses est nécessaire alors que l'éducation interculturelle met plus l'accent sur la laïcité et la citoyenneté démocratique. Une divergence quant à l'introduction des deux modèles dans l'espace scolaire est aussi relevée. Si la première est en général mise en œuvre après des initiatives bottom-up provenant de la société civile, des associations d'enseignant.e.s ou d'éducatrices/éducateurs et de parents (Khan, 2008), l'éducation interculturelle est plutôt introduite par une approche top-down. Les organisations internationales telles que le Conseil de l'Europe ou l'Organisation de coopération et de développement économiques (OCDE) convainquent les États de l'intérêt de promouvoir l'interculturel et l'intégration des élèves migrant.e.s à l'école (Besley \& Peters, 2012). Néanmoins, il faut se garder de conclure que cette divergence en matière de mise en ouvre proviendrait de la nature différente de l'éducation multiculturelle et interculturelle; les différences proviennent aussi de la gouvernance scolaire et son degré de centralisation. Dans les pays anglophones, le système éducatif est très décentralisé, et l'école est gouvernée par les commissions scolaires locales, sensibles à la société civile et aux attentes des parents et des éducatrices/éducateurs. Dans les pays francophones ou hispanophone d'Europe, la situation est contrastée. En France, l'école est une institution plutôt centralisée avec une marge de manœuvre locale faible à l'inverse de la Suisse, la Belgique ou l'Espagne dont la gouvernance est décentralisée. Ce qui amène les villes et les cantons en Suisse et les provinces autonomes en Espagne à avoir une influence importante sur la gestion de la diversité culturelle à l'école 
La littérature internationale est partagée au niveau de la différence réelle ou supposée entre modèle multiculturel et modèle interculturel. Joppke (2018) parle même d'une guerre des mots. Taylor (2012) estime que la différence n'est pas tant une question de politique concrète, mais plutôt l' "histoire que nous racontons à propos d'où nous venons et où nous allons. À certains égards, on pourrait dire que l'interculturalisme est plus approprié pour certains pays européens» (p. 413).

Cette distinction prend-elle sens en explorant les résultats de l'enquête PISA (Programme international pour le suivi des acquis des élèves)? Existe-t-il des liens objectivables entre l'ancrage plus ou moins marqué d'un pays dans l'un ou l'autre des modèles (multiculturel ou interculturel) et les résultats scolaires des élèves issus de la diversité culturelle (élèves immigré.e.s de première et de deuxième générations), et ainsi une distinction de l'effet des deux modèles d'intégration. Notre intention n'est pas d'établir un lien de causalité entre modèles d'intégration et différences de performance des jeunes de 15 ans dans les épreuves PISA selon leur statut (autochtone/migrant.e). Toutefois, l'attention à la diversité culturelle constituant un axe fort des politiques éducatives, il semble utile de comparer les performances des pays selon le modèle qu'ils adoptent, dans la lignée des travaux de Celeste, Baysu, Phalet, Meeussen, et Kende (2019) par exemple, qui ont étudié l'écart de performances entre jeunes appartenant à différents groupes ethniques (minoritaire, majoritaire) selon la politique de la diversité que les établissements fréquentés appliquent.

\section{PISA et diversité ethnoculturelle}

Il existe une littérature abondante sur l'enquête PISA, en particulier sur ses avantages et ses limites (Volante, 2018). Nous n'allons donc pas insister ici sur ses caractéristiques. Nous allons simplement rappeler qu'il s'agit d'une enquête créée au début des années 2000 par l'OCDE, et répandue à présent dans plus de 80 pays, dans le but de mesurer les performances scolaires des jeunes de 15 ans (fin de scolarité obligatoire) en lecture, mathématiques et sciences, et de faire des liens entre ses performances et des données plus contextuelles, notamment issues de questionnaires. Ce sont ce type de données qui sont particulièrement intéressantes lorsque nous nous intéressons aux problématiques ethnoculturelles.

En effet, les données produites par PISA explicitement sur la situation des élèves d'origine immigrée se sont de plus en plus complexifiées. Dans le cadre de l'enquête PISA 2015 que nous avons mobilisée pour cette article, l'OCDE distingue les élèves selon leur origine culturelle:

- Les élèves autochtones ou «élèves non issus de l'immigration» sont «les élèves dont la mère ou le père, ou les deux, sont nés dans le pays [...] où ils ont passé les épreuves PISA, que l'élève lui-même soit né dans ce pays [...] ou non».

- Les élèves immigré.e.s sont "les élèves dont la mère et le père sont nés dans un pays différent [... de celui] où l'élève a passé les épreuves PISA». Parmi 
les élèves issus de l'immigration, l'OCDE opère une distinction entre celles et ceux qui sont né.e.s dans le pays de l'évaluation et celles et ceux qui sont né.e.s dans un autre pays: les élèves immigré.e.s de la première génération sont «les élèves nés à l'étranger et dont les parents sont également nés à l'étranger»; les élèves immigré.e.s de la deuxième génération sont «les élève.e.s né.e.s dans le pays/l'économie où ils ont passé les épreuves PISA et dont les parents sont nés à l'étranger». (OCDE, 2018a, p. 23)

L'avantage des données de PISA 2015 est qu'elles distinguent les typologies d'élèves immigré.e.s (de la première et de la deuxième génération, statut socioéconomique, etc.). En revanche, selon les définitions de l'OCDE, les élèves dont les familles sont mixtes (les parents sont nés dans le pays et à l'étranger) sont considérés comme non issu.e.s de l'immigration. Bien que les données PISA ne soient pas la panacée pour considérer les enjeux complexes de l'interculturel et du multiculturel, elles ont l'avantage de donner des indications majeures sur les liens entre diversité ethnoculturelle et performances scolaires dans les pays sélectionnés.

Ainsi, de nombreuses études ont déjà mobilisé les données PISA, en particulier celles entre 2000 et 2009, pour faire des liens entre performances scolaires et origine ethnoculturelle. Le consensus général est que les résultats scolaires des élèves immigré.e.s sont inférieurs à ceux des autochtones. Les différences sont généralement liées au statut socio-économique (Entorf \& Minoiu, 2005; Gang \& Zimmermann, 2000). Le désavantage des immigré.e.s est en effet le plus faible dans les pays où leur composition sociale est la plus proche de celle des autochtones. Cattaneo et Wolter (2015) soulignent par exemple que la Suisse a radicalement changé sa politique migratoire au milieu des années 1990 - d'une politique d'accueil de populations non qualifiées à une politique favorisant l'immigration de migrant.e.s hautement qualifié.e.s. Les auteur.e.s constatent qu'environ $75 \%$ de l'augmentation (40 points) du score au PISA des élèves immigrant.e.s de première génération est attribuable à des changements dans les antécédents individuels de leurs parents et à une meilleure composition scolaire.

Parmi les mesures éducatives facilitant l'intégration des élèves immigré.e.s et de meilleures performances scolaires, les compétences linguistiques sont un facteur déterminant à considérer. Dans l'ensemble des pays, pour les élèves issu.e.s de l'immigration, la langue parlée à la maison est cruciale. Dustmann, Frattini et Lanzara (2012) comparent le niveau de scolarité des immigrant.e.s de deuxième génération à celui des enfants né.e.s de parents autochtones dans plusieurs pays de l'OCDE. Le désavantage des enfants immigré.e.s diminue, voire disparaît pour certains pays, une fois que l'on s'appuie sur les caractéristiques de l'origine parentale. Une langue étrangère parlée à la maison est le facteur le plus important associé à l'écart de performance scolaire. Au Royaume-Uni et aux États-Unis, les compétences linguistiques semblent être le principal obstacle pour les élèves immigré.e.s (Schnepf, 2007). La littérature préconise donc de mettre l'accent sur les compétences linguistiques au tout début de l'enfance (Cobb-Clark, Sinning, \& 
Stillman, 2011). Que ce soit pour les compétences linguistiques ou pour l'origine socioéconomique, Schnepf (2007) précise qu'au niveau individuel ce sont des déterminants importants, mais pas exclusifs, des écarts de performances scolaires.

La ségrégation ou différenciation précoce des élèves peut également avoir un impact négatif. En Nouvelle-Zélande, les résultats des immigré.e.s de deuxième génération sont pires que ceux de la première génération; constat en partie expliqué par la répartition inégale des élèves immigré.e-s entre les écoles (Schnepf, 2007). En Suisse, d'après les données PISA, les cantons où la relégation des élèves de deuxième génération dans les filières les moins exigeantes est la plus marquée sont aussi ceux où les inégalités d'acquis par rapport aux natives et natifs sont les plus importantes (Felouzis, Charmillot, \& Fouquet-Chauprade, 2016).

Dans certains pays, ces différents facteurs contribuent grandement à l'écart de réussite, dans d'autres, seulement de manière marginale. D'ailleurs, certaines études ont distingué des types de pays, selon les performances scolaires des élèves. Schnepf (2007) montre que dix pays à forte immigration examinés peuvent être divisés en deux groupes: les pays anglophones où le désavantage des immigré.e.s est plus faible et les pays d'Europe continentale où ils présentent un désavantage éducatif élevé.

Notre analyse vient ainsi compléter toute cette littérature en mobilisant des données plus récentes de PISA, lesquelles utilisent des indicateurs plus précis pour identifier les enjeux liés aux élèves immigré.e-s. Par ailleurs, nous allons nous concentrer sur des pays ayant adopté l'interculturalisme (Espagne, France, Suisse romande) avec des pays privilégiant le multiculturalisme (Canada anglophone, États-Unis, Royaume-Uni) pour tenter de dégager des conclusions sur les modèles d'intégration et leurs liens avec les performances scolaires.

\section{PISA 2015: Liens entre performances scolaires et L'origine culturelle}

Cette analyse porte sur des statistiques existantes: les données de PISA, librement accessibles sur le site internet de l'OCDE. À partir de la multitude de données disponibles - 34 États membres de l'OCDE, ainsi que des pays partenaires tels que la Russie et le Brésil - et sur les performances scolaires ainsi des éléments contextuels variés, nous avons retenu celles concernant les six pays de notre étude et les élèves immigré.e.s. Les pays ont été subdivisés pour les besoins de l'analyse en deux sous-groupes: groupe 1 - pays «interculturels»; groupe 2 - pays «multiculturels». Des tableurs ont été exportés pour l'analyse et l'élaboration des graphiques ci-dessous.

\section{Les élèves immigré-e-s dans PISA 2015}

Avant toute analyse, le profil des élèves des six pays doit être établi. Ce profil correspond à celui des élèves ayant passé le test PISA 2015; s'ils montrent 
une tendance, ils ne sont pas complètement représentatifs de l'ensemble de la population des pays étudiés.

Concernant la part d'élèves immigré.e·s ayant participéà PISA 2015, la Suisse, le Canada et les États-Unis sortent du lot avec plus de $20 \%$. Les autres pays se situent entre 11 et $17 \%$. Au sein de la catégorie, on trouve des élèves immigré.e.s de première et de deuxième génération. En France, en Suisse et aux États-Unis, la deuxième génération est plus représentée. En Espagne, ce sont les élèves de première génération qui sont plus nombreux. Au Canada et au Royaume-Uni, la part est à peu près égale.

À cette part d'élèves immigré.e.s, nous devons ajouter d'autres dimensions, à savoir, en premier lieu, le statut socio-économique. Les pays que nous considérons comme «multiculturels» sont ceux qui sélectionnent particulièrement les populations migrantes: globalement, les populations émigrantes qui comptent la plus forte proportion de personnes ayant un niveau d'études supérieures sont principalement installées aux États-Unis, au Canada et au Royaume-Uni. Dans PISA 2015, le Canada a plus de la moitié des élèves immigré.e.s ayant participé au test qui ont un statut socioéconomique avantageux, ce qui n'est le cas dans aucun autre pays de cette étude. Les élèves favorisé.e.s sont défini.e.s comme les élèves dont la valeur sur l'indice PISA de statut économique, social et culturel (ESCS) se situe dans le quart supérieur des élèves de tous les pays et toutes les économies de l'OCDE. En Suisse, aux États-Unis et au Royaume-Uni, ces élèves favorisé.e.s sont plus nombreuses/nombreux que les élèves défavorisés.e. En France, la part est à peu près égale. En Espagne, il y a davantage d'élèves défavorisé.e.s. À part au Canada, les élèves se situant dans les quarts les plus élevés et les moins élevés ne sont pas conséquents (en dessous de 7,2 \%).

Si nous regardons en détail cet indice ESCS pour PISA 2015, à part le Canada où les élèves immigré.e.s sont dans une situation avantageuse, dans les autres contextes, ces élèves sont largement défavorisé.e.s. Les écarts de statuts sont frappants en France, en Suisse et aux États-Unis (graphique 1).

D'autres indicateurs permettent de mieux saisir le profil des élèves immigré.e.s et de confirmer les constats précédents. Pour les élèves dont au moins un parent a un niveau de scolarisation similaire à la moyenne des parents d'élèves autochtones dans le pays d'accueil, ce sont le Canada et le Royaume-Uni qui sortent du lot puisqu'entre 72 et $74 \%$ des parents d'élèves immigré.e.s de la première génération sont concernés. La moyenne de l'OCDE est au-dessus des $50 \%$ alors que les autres pays de notre étude sont en-dessous (graphique 2). 
1,00

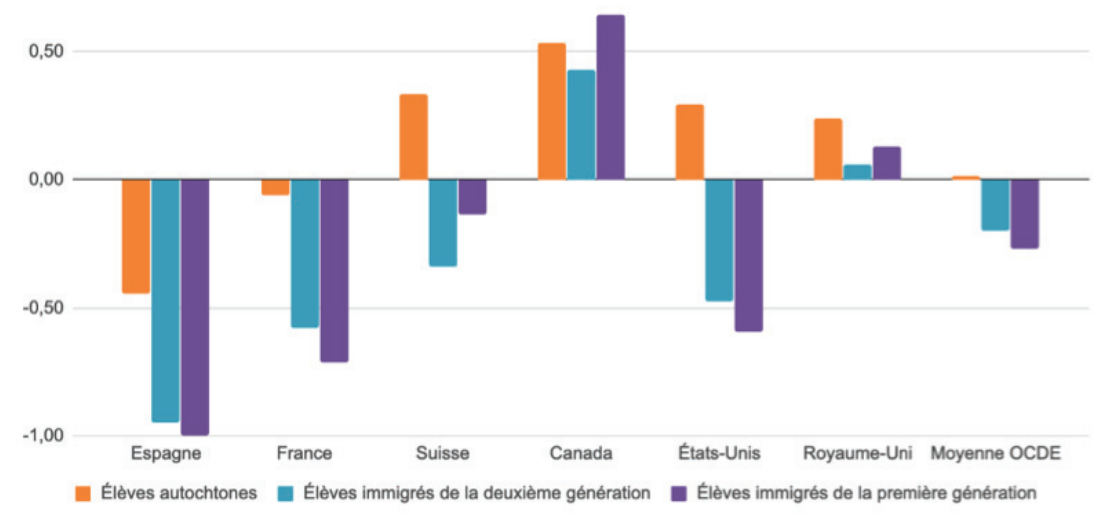

Graphique 1. Indice PISA de statut économique, social et culturel (ESCS). Source: OCDE (2016)

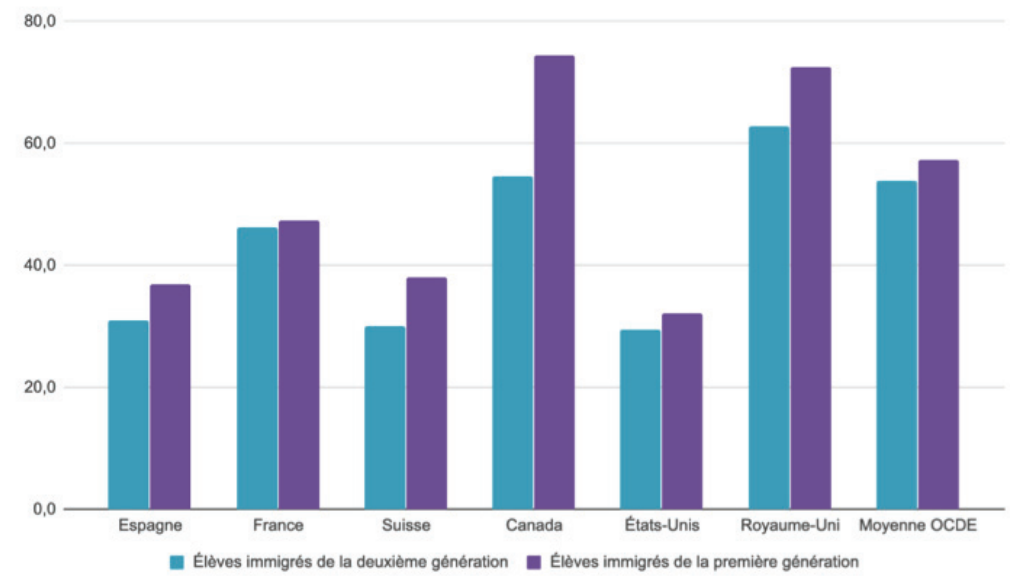

Graphique 2. Pourcentage d'élèves dont les parents ont un niveau de scolarisation similaire à la moyenne des parents d'élèves autochtones. Source: OCDE (2016)

En revanche, sur le fait de parler une autre langue à la maison, les six pays se retrouvent tous dans une situation similaire à celle de la moyenne de l'OCDE. En effet, les élèves autochtones parlent pratiquement toutes et tous la langue du test, donc généralement celle de l'école, à la maison alors que nombreuses/ nombreux sont celles et ceux à parler une langue différente parmi les élèves d'origine immigrée, avec parfois une distinction entre la première et la deuxième génération. 
Performances scolaires et élèves immigré-e-s

En matière de performances générales dans les trois domaines évalués par PISA, toutes origines culturelles confondues, les deux groupes de pays se situent près dans la moyenne de l'OCDE, le Canada ayant toutefois des performances plus élevées (graphique 3).

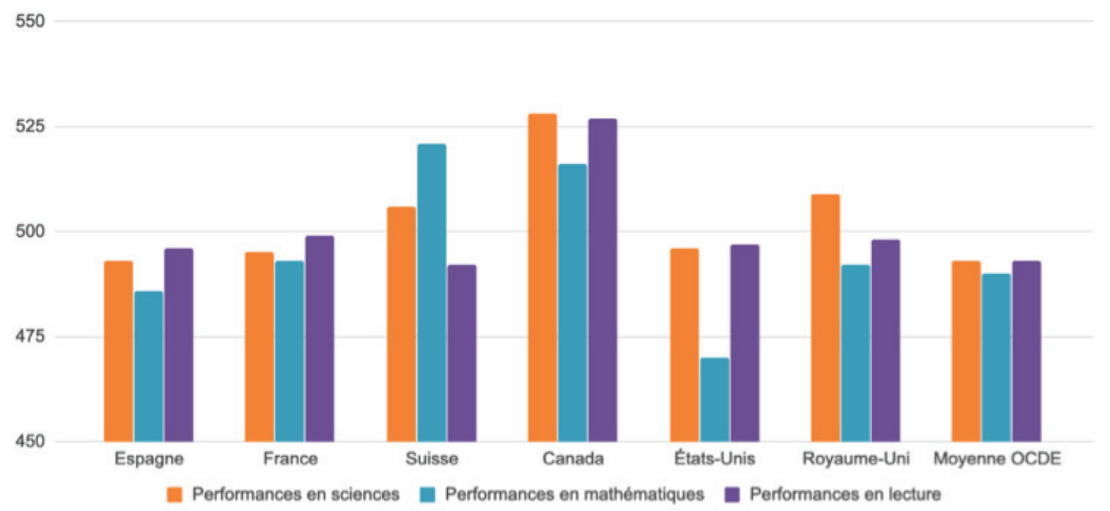

Graphique 3. Performances générales des élèves. Source: OCDE (2016)

Ce constat général concerne-t-il l'ensemble des élèves, d'origine non immigrée et immigrée? En prenant cette fois en considération l'origine ethnoculturelle, nous remarquons que les pays sélectionnés ont une tendance proche de celle de la moyenne de l'OCDE, à savoir que les élèves autochtones ont de meilleures performances que les élèves d'origine immigrée, avec souvent des grands écarts (Espagne, France, Suisse et États-Unis). À l'intérieur de la catégorie des élèves immigré.e.s, celles et ceux de la deuxième génération font mieux que celles et ceux de première génération. Une exception nette correspond aux résultats du Canada où l'ensemble des élèves ont de très bonnes performances quelle que soit leur origine culturelle. Les élèves immigré.e.s font même légèrement mieux. Au Royaume-Uni également, il n'y a pas beaucoup d'écarts entre l'ensemble des élèves. Pour illustrer notre propos, nous avons retenu les écarts de performance en sciences, qui était le domaine principal testé par PISA 2015, tout en notant que les tendances sont relativement similaires dans les autres disciplines (graphique 4). 
550

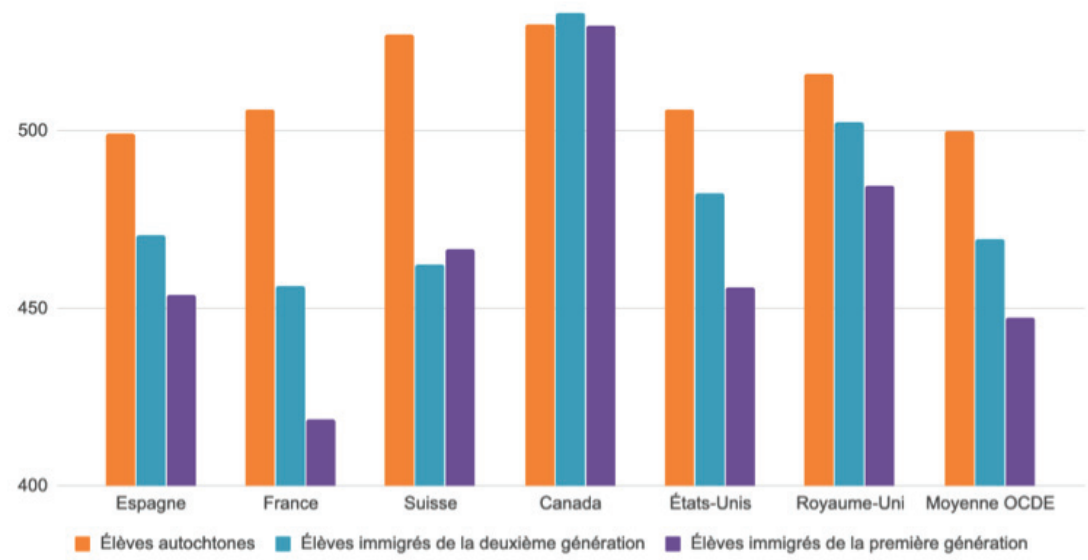

Graphique 4. Ecarts de performance en sciences entre les élèves autochtones et les élèves immigré.e.s. Source: OCDE (2016)

Ainsi, la sous-performance scolaire est commune à la plupart des élèves immigré.e.s, mais en particulier à celles et ceux de la première génération. Dans l'ensemble des pays ayant passé PISA 2015, 51 \% des élèves immigré.e.s de première génération n'ont pas atteint les niveaux de compétences de base en lecture, en mathématiques et en sciences, contre $28 \%$ des élèves autochtones. Si nous regardons la situation spécifique des six pays, mis à part le Canada, ils se situent autour de la moyenne de l'OCDE en ce qui concerne le pourcentage d'élèves très peu performant.e.s en sciences (score inférieur au niveau 2) ${ }^{1}$. Les élèves autochtones représentent entre $10 \%$ et $18 \%$ de cette catégorie alors que les élèves immigré.e.s de la deuxième génération entre $17 \%$ et $35 \%$, et celles et ceux de la première génération entre $25 \%$ et $35 \%$. Dans ce dernier cas, la France sort du lot avec $50 \%$ des élèves de la première génération qui ont des performances inférieures au niveau 2. Ce qui est intéressant avec le Canada, c'est que l'ensemble des élèves (autochtones et immigré·e-s) ont des résultats relativement similaires (graphique 5).

Pour ce qui est du pourcentage d'élèves les plus performant.e.s en sciences (niveau 5 et plus), une fois de plus, le Canada mis de côté, nous constatons que les élèves autochtones sont dans une situation plus favorable que les élèves immigré.e.s, avec des particularités contextuelles. C'est en France et en Suisse que ces écarts sont les plus importants. D’ailleurs, dans ce dernier pays, les élèves immigré.e.s de première génération ont de meilleurs résultats que celles et ceux de deuxième génération. À noter également qu'au Canada, les élèves immigré·e.s font légèrement mieux que les élèves autochtones, sachant que dans tous les cas, les élèves sont plus performant.e.s que dans l'ensemble des autres pays (graphique 6). 
60,0

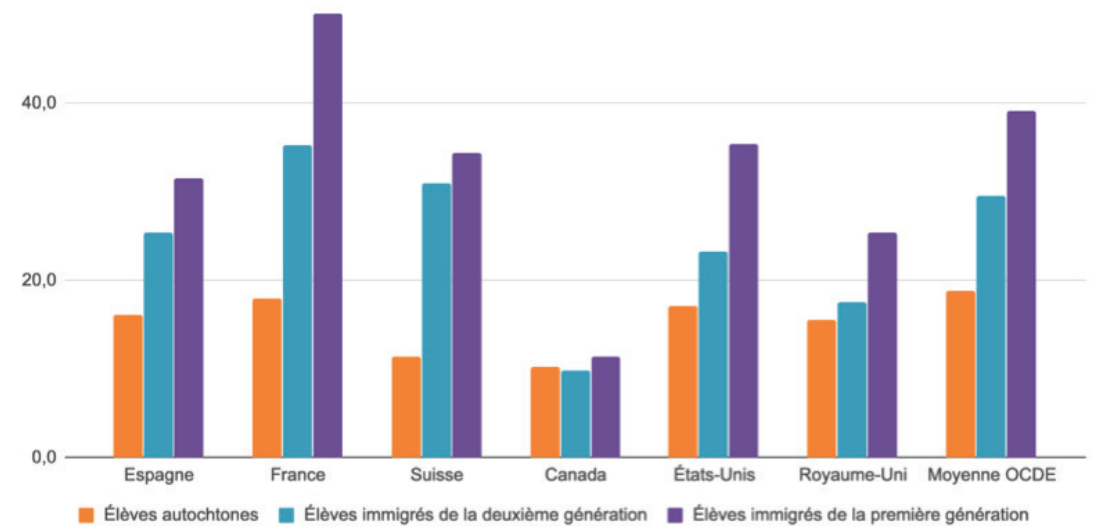

Graphique 5. Pourcentage d'élèves peu performant.e.s en sciences (inférieur au nivean 2). Source: OCDE (2016)

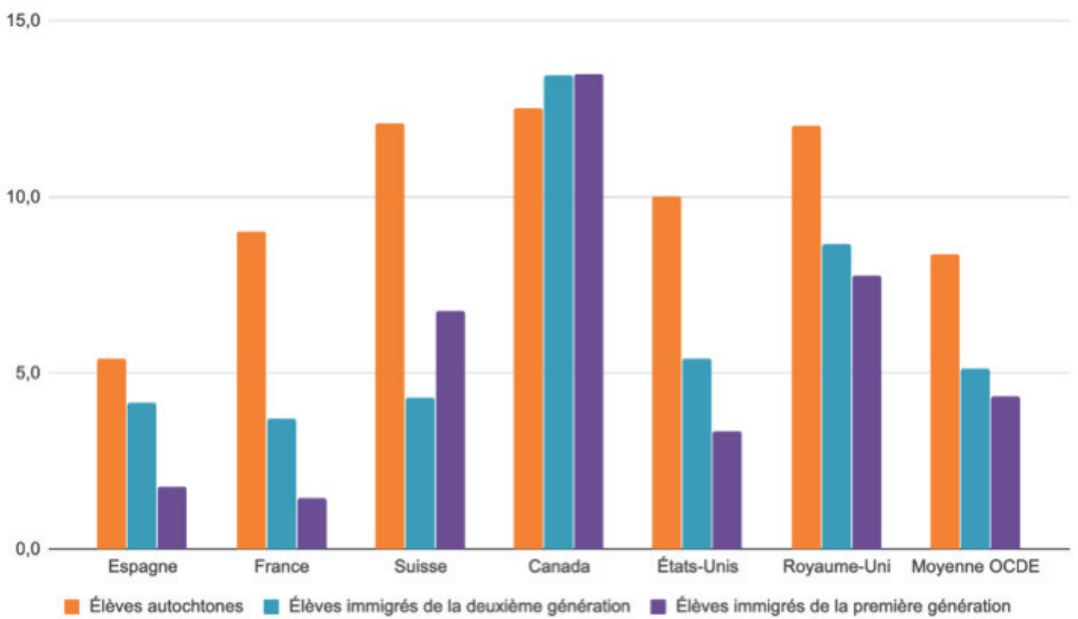

Graphique 6. Pourcentage d'élèves très performant.e.s en sciences (niveau 5 ou supérieur). Source: OCDE (2016)

\section{Dimensions socioéconomiques et linguistiques}

En considérant le statut socio-économique des élèves autochtones et élèves immigré.e.s les écarts de performance mentionnés plus haut demeurent mais s'amenuisent. Ainsi c'est en France, et plus encore, aux États-Unis que le statut socio-économique joue un rôle clé. Dans ce dernier pays, en lecture et en mathé- 
matiques, si on prend en compte ce statut, les élèves immigré.e.s font aussi bien que les élèves autochtones (graphique 7).

75

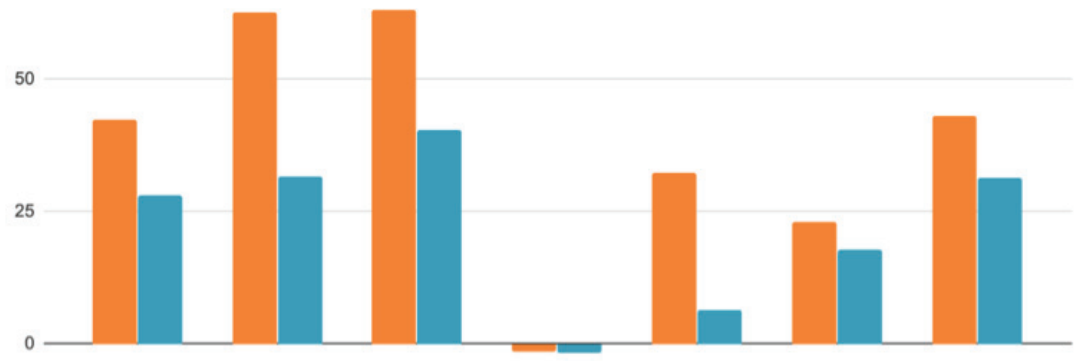

$-25$

Espagne France Suisse Canada Etats-Unis Royaume-Uni Moyenne OCDE
Avant avoir pris en compte le statut socio-économique $\quad$ Après avoir pris en compte le statut socio-économique

Graphique 7. Écarts de performance en sciences entre les élèves autochtones et les élèves immigré.e.s, et statut socio-économique. Source: OCDE (2016)

Si nous prenons les données de PISA 2015 qui établissent une corrélation entre les performances scolaires et la langue parlée à la maison, nous voyons que pour les pays du groupe 1, même si les élèves immigré.e.s parlent la langue de scolarisation à la maison, elles/ils ont des résultats inférieurs à celles et ceux des élèves autochtones. À l'inverse, dans les pays du groupe 2, les performances sont quasi identiques que l'on soit d'origine immigrée ou non. Quant aux élèves ne parlant pas la langue du test à la maison, dans tous les cas, quelle que soit leur origine, elles/ils ont tendance à avoir des résultats plus faibles que celles et ceux parlant la langue du test. Ce qui est intéressant de noter, c'est qu'en France, au Canada, aux États-Unis et au Royaume-Unis, ce sont les élèves immigré.e.s qui ont de meilleures performances lorsqu'ils parlent une autre langue à la maison. C'est particulièrement frappant pour les performances en lecture (graphique 8).

Là où les chiffres sont percutants, c'est lorsque l'on considère le statut socioéconomique cumulé avec la langue parlée à la maison. Dans ce cas, il est clair que les différences sont marquées entre élèves autochtones et élèves immigré.e.s en défaveur de ces derniers, sauf au Canada, mais en particulier dans les pays du groupe 1. Lorsqu'on ajoute la dimension socio-économique, à part en Espagne, ces différences sont nettement moins grandes. C'est flagrant pour le cas de la France et de la Suisse. Aux États-Unis, les élèves immigré.e.s et autochtones ayant un profil socioéconomique similaire ont des chances égales d'atteindre les compétences de base (graphique 9). Ainsi, le désavantage socioéconomique et les barrières linguistiques sont deux des principaux obstacles à la réussite scolaire des élèves immigré.e.s. 
550

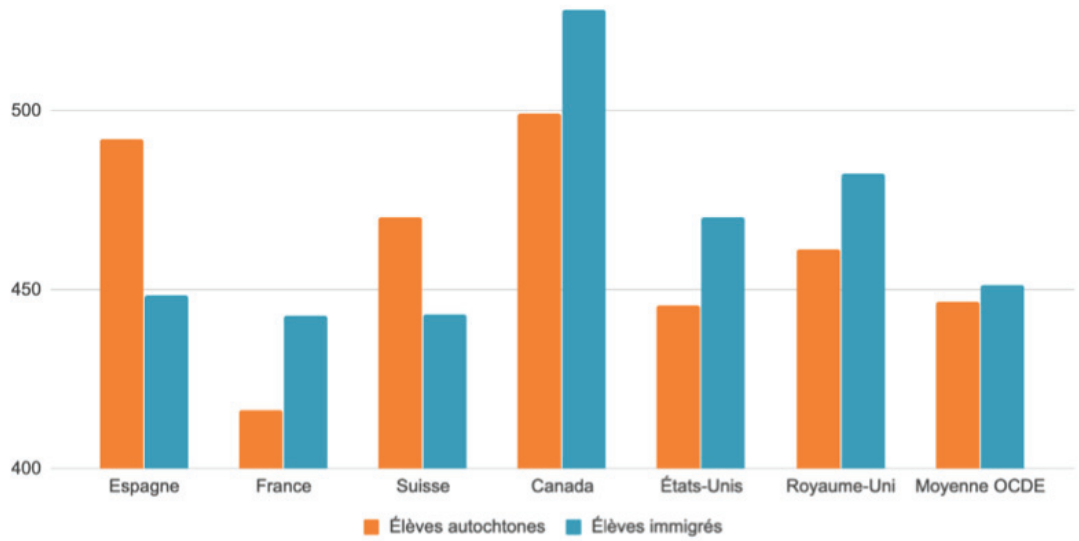

Graphique 8. Écarts de performances en lecture pour les élèves qui parlent principalement une autre langue à la maison Source: OCDE (2016)

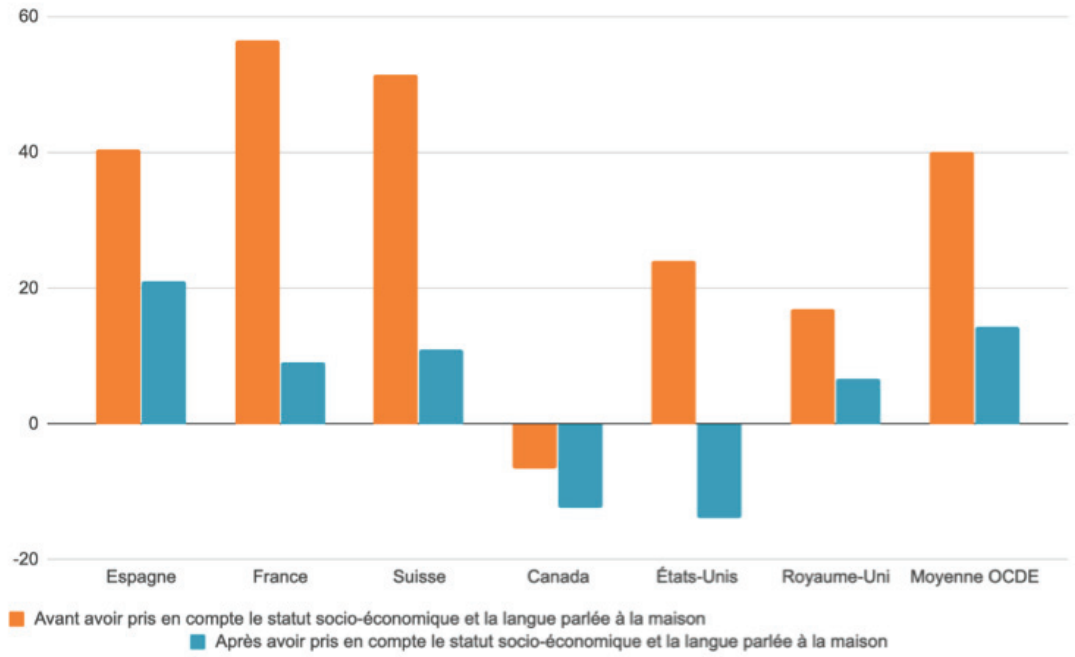

Graphique 9. Écarts de performance en lecture liés à l'origine culturelle, au statut socio-économique et à la langue parlée à la maison. Source: OCDE (2016)

\section{Dimensions structurelles}

Au-delà des facteurs socioéconomiques et linguistiques, les données de PISA 2015 apportent de nouveaux indicateurs du point de vue structurel. Parmi eux, nous pouvons évoquer le taux de concentration des élèves immigré.e.s dans les mêmes 
écoles. Il est intéressant d'observer que pour les pays du groupe 2, le pourcentage d'élèves, immigré.e.s et non immigré.e.s, qui devraient être déplacé.e.s d'une école à l'autre afin que toutes les écoles du pays aient un pourcentage identique d'élèves immigré.e.s, est plus élevé. Ainsi, une plus forte proportion d'élèves immigré.e.s dans les établissements d'enseignement n'est pas toujours associée à une faible performance des élèves. Ou alors, cette corrélation s'explique principalement par la concentration d'élèves défavorisé.e.s dans ces établissements (graphique 10).

30,0

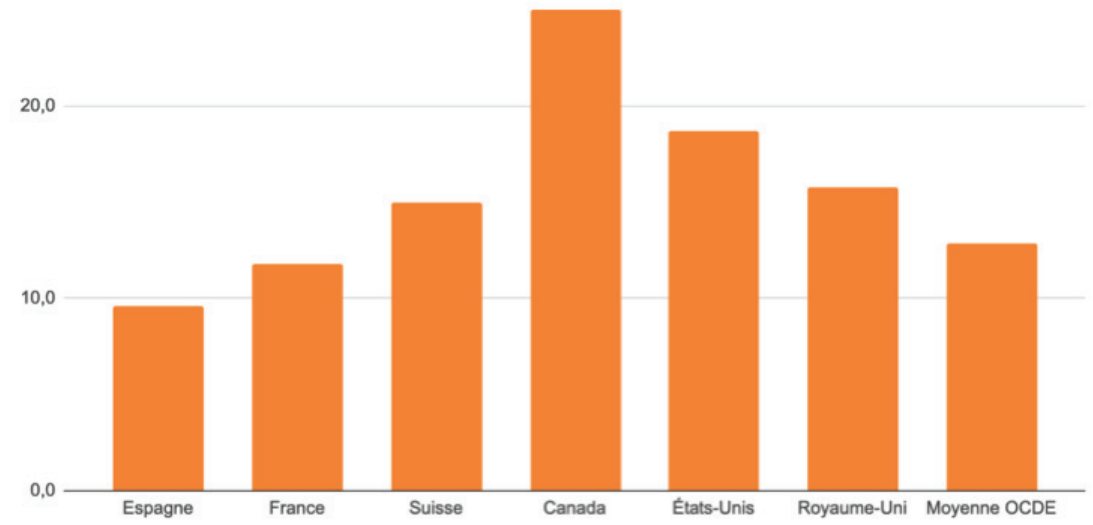

Graphique 10. Indice de concentration des élèves immigré.e.s dans les écoles. Source: OCDE (2016)

Il est difficile de tirer des conclusions claires à partir des ressources éducatives disponibles entre les écoles avec des concentrations faibles et élevées d'élèves immigré.e.s. En Espagne, et dans une moindre mesure en France et au Canada, les écoles avec des concentrations élevées d'élèves immigré.e.s sont moins bien dotées en infrastructures et en matériel pédagogique. Ces écoles dans les autres pays sont identiquement ou mieux pourvues. En Espagne, et dans une moindre mesure en Suisse et au Royaume-Uni, les écoles avec des concentrations élevées d'élèves immigré.e.s sont moins bien dotées en enseignant.e.s qualifié.e.s, contrairement aux autres pays.

Pour finir, nous souhaitons mettre en évidence les indicateurs du bien-être des élèves que propose pour la première fois l'enquête PISA 2015 à travers des variables telles que l'anxiété, l'intérêt ou la motivation à l'idée de réussir. Ces données se fondent généralement sur les déclarations des élèves. Ici, nous regardons la différence de pourcentage d'élèves se disant d'accord/tout à fait d'accord avec l'affirmation "Je me sens chez moi à l'école» entre les élèves autoch- 
tones et les élèves immigré.e.s de première génération. Nous constatons une différence entre les pays des groupes 1 et 2 . En effet, les immigré.e.s se sentent moins chez eux à l'école en Espagne, en France et en Suisse. C'est le contraire qui est vrai au Canada, au Royaume-Uni et aux États-Unis.

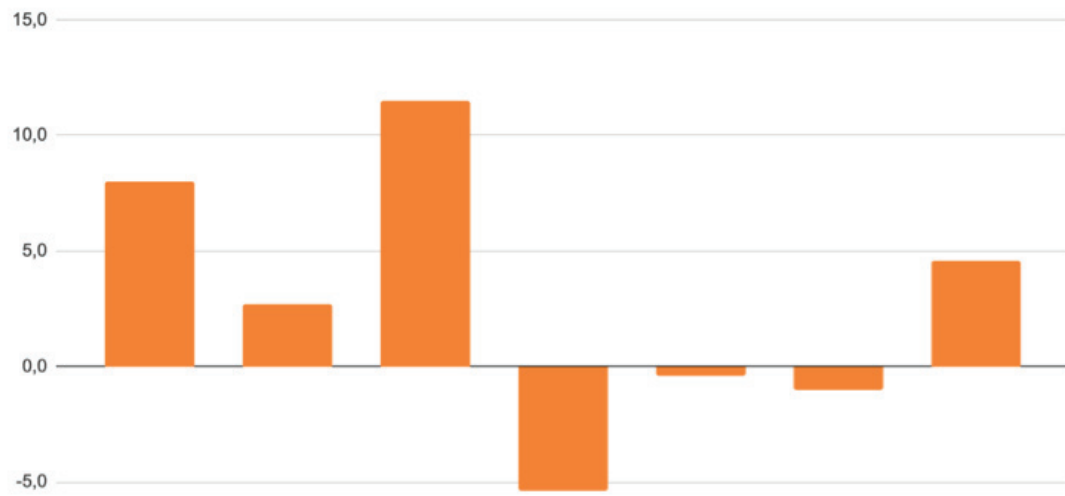

$-10,0$ Espagne Suisse Canada États-Unis Royaume-Uni Moyenne OCDE

Graphique 11. Différence de pourcentage d'élèves se disant d'accordltout à fait d'accord avec l'affirmation "Je me sens chez moi à l'école» entre les élèves autochtones et les élèves immigré.e.s de la première génération. Source: OCDE (2018b)

\section{Discussion: un modèle d'intégration se reflète-t-il dans les résultats de l'enquête PISA?}

Quelles leçons peuvent être tirées de cette analyse des données de l'enquête PISA 2015, questionnant les liens potentiels entre modèles d'intégration (interculturel/multiculturel) et performances scolaires? Un aperçu de la situation générale en termes de performances (tous indicateurs confondus) des six pays choisis indique que dans les deux groupes, il y a une variabilité de situations. Dans le groupe 1 («interculturel»), il y a des pays qui font mieux que d'autres, de même dans le groupe 2 («multiculturel»). Ils se situent de part et d'autre de la moyenne de l'OCDE.

Toutefois, en portant une attention aux écarts de performances scolaires entre élèves autochtones et immigré.e.s (première et deuxième génération), ces écarts sont moins marqués dans les pays du groupe 2 que dans ceux du groupe 1 . Ce résultat est intéressant pour plusieurs raisons. Tout d'abord, il invalide partiellement l'hypothèse avancée par les auteur.e.s francophones sur la supériorité du 
modèle interculturel pour la réussite scolaire des élèves migrant.e.s. Ensuite, nous observons une hétérogénéité au niveau des écarts entre autochtones et migrant.e.s (première et deuxième génération) parmi les pays du groupe 2 (modèle multiculturel). Le Canada, même si les données ne différencient pas les parties anglophone et francophone du pays, se distingue par l'écart le plus faible et sa capacité de permettre aux élèves d'origine migrante d'avoir des performances élevées dans le test PISA. Nous constatons que c'est le seul pays qui a adopté au niveau le plus élevé de l'État la politique du multiculturalisme. Le Royaume-Uni a certes des écarts plus importants, mais semble aussi favorable pour de bonnes performances des élèves issu.e.s de la migration. La situation des États-Unis est plus proche de celle des pays d'Europe continentale, mais appelle une prudence en matière d'interprétation. Contrairement aux cinq autres pays de notre échantillon, les États-Unis comportent un pourcentage important d'élèves issu.e.s de la diversité ethnoculturelle interne (Afro-américains et Chicanos), qui ne peuvent pas être considéré.e.s comme issus de la migration. Par ailleurs, cette convergence des États-Unis avec l'Europe continentale au niveau des performances scolaires des élèves migrant.e.s a été déjà mise en évidence par Alba et Silberman (2009) sur la base d'une comparaison entre Maghrébins en France et Mexicains aux ÉtatsUnis. Malgré les différences apparentes entre ces deux systèmes éducatifs, la ségrégation résidentielle et le suivi pédagogique différencié produisent les mêmes résultats, qui reflètent la volonté des parents autochtones de classe moyenne de préserver leur statut privilégié en contrecarrant les efforts visant à rendre le système éducatif plus ouvert et équitable.

Concernant les systèmes interculturels, la France, la Suisse et l'Espagne se distinguent par des écarts importants au niveau des performances des élèves entre autochtones et migrant.e.s. Ce résultat est d'autant plus préoccupant que le niveau de ségrégation résidentielle est beaucoup plus important aux États-Unis par rapport à ces trois pays.

Il est bien entendu difficile, sur la seule base du modèle implicite ou explicite d'intégration (interculturel ou multiculturel), d'expliquer les résultats d'une enquête aussi complexe que PISA. D'autres éléments permettent de nuancer l'interprétation et de ne pas surestimer l'importance du modèle national d'intégration. En effet, il faut noter que la gestion des systèmes éducatifs dans les six pays est différente, et en particulier la familiarité et la pression des tests standardisés du type PISA est plus ancienne et plus forte dans les pays anglo-saxons. D'autres éléments peuvent également expliquer la variabilité des écarts entre autochtones et migrant.e.s comme la taille du secteur privé et son accessibilité ou l'existence ou pas de carte scolaire (Dronkers \& Avram, 2009).

Par ailleurs, les travaux d'Ogbu $(1987,1991)$ ont mis en évidence que la manière conformiste (réussite scolaire) ou oppositionnelle que les minorités ethnoculturelles et les migrant.e.s adoptent dans le système scolaire va dépendre de deux éléments: a) les termes initiaux d'incorporation des minorités dans l'État-Nation (par exemple esclavagisme, conquête, colonisation) et b) le 
traitement que le système scolaire réserve aux histoires, langues et cultures des minorités ethnoculturelles. Les écarts importants constatés en France peuvent être expliqués par la provenance de l'ancien empire colonial d'une partie importante des jeunes de 15 ans (Akkari, 2001).

Les barrières linguistiques et un statut socio-économique relativement défavorisé sont des facteurs de risque qui augmentent considérablement la vulnérabilité des élèves immigré.e.s, en particulier celles et ceux de première génération qui s'établissent dans un nouveau pays. Autrement dit, une piste serait de soutenir la population d'origine immigrée et de favoriser son insertion socio-économique. Il serait également préférable d'agir sur les causes des écarts de performances scolaires liés aux statuts socio-économiques. Cela nécessite de proposer des politiques sociales qui dépassent le seul champ de l'éducation, bien qu'il faille s'interroger sur le rôle de l'école en tant que lieu de promotion sociale, et non de reproduction des inégalités sociales. Dans le même ordre idée, les résultats interpellent dans certains contextes sur des inégalités en matière de disponibilité d'enseignant.e.s qualifié.e.s et/ou de ressources pédagogiques au détriment des élèves immigré.e.s ou socialement défavorisé.e.s (OECD, 2018a; Rangvid, 2007). Enfin, l'école doit être en mesure d'accueillir toutes et tous les élèves quel que soit leur niveau socioéconomique, et avoir pour visée l'ouverture interculturelle (valorisation de la diversité, sentiment d'appartenance, etc.) afin de donner confiance aux élèves issu.e.s de l'immigration en valorisant toutes leurs potentialités (Ham, Yang, \& Cha, 2017).

Sur ce dernier aspect, les données ont également révélé que les élèves d'origine immigrée manquaient souvent d'un sentiment d'appartenance à leur communauté scolaire et étaient plus susceptibles d'être atteints d'anxiété liée au travail scolaire (Benimmas, Boutouchent, Kamano, \& Bourque, 2014; Schachner, He, Heizmann, \& Van de Vijver, 2017). Il est donc recommandé d'accorder un soutien supplémentaire aux élèves et aux écoles défavorisé.e.s, de mettre en œuvre des programmes efficaces de lutte contre l'intimidation, d'assurer la disponibilité et la participation aux activités parascolaires, de promouvoir la participation des parents aux activités scolaires (LKenfack, 2017). Les enseignant.e.s sont sûrement des actrices/acteurs clés du bien-être des élèves immigré.e.s pour assurer leur bien-être influençant leurs performances scolaires. Les politiques éducatives peuvent favoriser un corps enseignant soucieux de la diversité pouvant soutenir toutes et tous les apprenant.e.s. De nombreuses/nombreux élèves immigré.e.s ont le sentiment d'être traité.e.s injustement par leurs enseignant.e.s, ce qui suggère que ces derniers peuvent ne pas savoir comment soutenir efficacement leurs élèves à faibles performances scolaires (Fine-Davis \& Faas, 2014; Thijs \& Fleischmann, 2015). Beaucoup d'enseignant.e.s le reconnaissent puisque, que dans de nombreux systèmes éducatifs, ils déclarent ressentir le besoin de développer leur capacité à gérer des classes multiculturelles (OECD, 2018b). Ainsi, les politiques doivent également investir dans le développement de programmes conçus pour aider les enseignant.e.s à enseigner dans différentes 
classes et à améliorer la qualité des modules de formation existants. Cela permettrait entre autres d'aider les enseignant.e.s à prendre conscience, et même de valoriser, les antécédents et la situation de leurs élèves, et d'être conscient·e·s du large éventail de mécanismes par lesquels différentes expériences de migration peuvent influer sur les performances scolaires.

En définitive, l'enquête comparative internationale PISA représente depuis deux décennies une référence pour le débat sur les politiques éducatives nationales dans les pays de l'OCDE. De nombreuses recherches tentent d'identifier les raisons des bonnes performances de certains pays. Dans cet article, nous avons exploré, malgré la complexité de la question, le rôle du modèle d'intégration des migrant.e.s dans les performances de six pays de l'OCDE. L'hypothèse de la supériorité du modèle interculturel ne semble pas plausible. Il existe même des indices pour soutenir une plus grande capacité d'intégration des élèves migrant.e.s du modèle multiculturel, notamment au Canada.

\section{Note}

1 Les scores de PISA à l'échelle d'un pays sont gradués de manière à ce que la moyenne arithmétique de tous les pays soit de 500 avec un écart type de 100.40 points PISA représente une année de scolarité. À partir de ce critère, les scores sont répartis en niveau:

- Au-dessous du niveau 1: score inférieur ou égal à 357,77

- Niveau 1: score supérieur à 357,77 et inférieur ou égal à 420,07

- Niveau 2: score supérieur à 420,07 et inférieur ou égal à 482,38

- Niveau 3: score supérieur à 482,38 et inférieur ou égal à 544,68

- Niveau 4: score supérieur à 544,68 et inférieur ou égal à 606,99

- Niveau 5: score supérieur à 606,99 et inférieur ou égal à 669,30

- Niveau 6: score supérieur à 669,30

\section{Références}

Abdallah-Pretceille, M. (2011). La pédagogie interculturelle: entre multiculturalisme et individualisme. Linguarum Arena: Revista do Programa Doutoral em Didáctica de Linguas da Universidade do Porto, 2, 91-101.

Akkari, A. (2001). Les jeunes d'origine maghrébine en France: Les limites de l'intégration par l'école. Revue électronique de Sociologie, 3(8). Repéré à http://critique.ovh.org/

Alba, R., \& Silberman, R. (2009). The children of immigrants and host-society educational systems: Mexicans in the United States and North Africans in France. Teachers College Record, 111(6), 1444-1475.

Ashcroft, R. T., \& Bevir, M. (2018). Multiculturalism in contemporary Britain: policy, law and theory. Critical Review of International Social and Political Philosophy, 21(1), 1-21.

Auger, N. (2007). Enseignement des langues d'origine et apprentissage du français: vers une pédagogie de l'inclusion. Le français aujourd'hui, (3), 76-83.

Banks, J. A. (1993). Multicultural education: Historical development, dimensions, and practice. Review of research in education, 19(1), 3-49.

Benimmas, A., Boutouchent, F., Kamano, L., \& Bourque, J. (2014). Le sentiment d'appartenance chez les immigrants francophones du Nouveau-Brunswick. Canadian Ethnic Studies, 46(1), 87-120.

Besley, T., \& Peters, M. A. (Éds.). (2012). Interculturalism, education and dialogue. New York, 
NY: Peter Lang.

Brahm Levey, G. (2012). Interculturalism vs. multiculturalism: a distinction without a difference? Journal of intercultural studies, 33(2), 217-224.

Cattaneo, M., \& Wolter, S. (2015). Better migrants, better PISA results: Findings from a natural experiment. IZA Journal of Migration, 4(1), 18.

Catarci, M., \& Fiorucci, M. (2016). Intercultural Education in the European Context. London, United Kingdom: Routledge.

Celeste, L., Baysu, G., Phalet, K., Meeussen, L., \& Kende, J. (2019). Can school diversity policies reduce belonging and achievement gaps between minority and majority youth? Multiculturalism, colorblindness, and assimilationism assessed. Personality and Social Psychology Bulletin, 45(11), 1603-1618.

Cobb-Clark, D., Sinning, M., \& Stillman, S. (2011). Migrant youths' Educational achievement: The role of institution. The Annals of the American Academy of Political and Social Science, 243, 18-45.

Dasen, P. R., \& Perregaux, C. (2000). Pourquoi des approches interculturelles en sciences de l'éducation (Vol. 3). Bruxelles, Belgique: De Boeck Supérieur.

Day, R. J. (2000). Multiculturalism and the history of Canadian diversity. Toronto, Canada: University of Toronto Press.

Dronkers, J., \& Avram, S. (2009). Choice and Effectiveness of Private and Public Schools in seven countries. A reanalysis of three PISA dat sets. Zeitschrift für Pädagogik, 55(6), 895-909.

Dustmann, C., Frattini, T., \& Lanzara, G. (2012). Educational achievement of second generation immigrants: an international comparison. Economic Policy, 27(69), 143-185.

Entorf, H., \& Minoiu, N. (2005). What a difference immigration policy makes: A comparison of PISA scores in Europe and traditional countries of immigration. German Economic Review, 6(3), 355376.

Felouzis, G., Charmillot, S., \& Fouquet-Chauprade, B. (2016). Les élèves de deuxième génération en Suisse: modes d'intégration scolaire et compétences acquises dans 13 systèmes éducatifs cantonaux. Swiss Journal of Sociology, 42(2), 218-244.

Ferrer, F., \& Akkari, A. (2000). La educación intercultural en España y en Suiza: un enfoque comparativo. Revista española de educación comparada, 6, 285-316.

Fine-Davis, M., \& Faas, D. (2014). Equality and diversity in the classroom: A comparison of students' and teachers' attitudes in six European countries. Social Indicators Research, 119(3), 1319-1334.

Gang, I., \& Zimmermann, K. (2000). Is child like parent? Educational attainment and ethnic origin. Journal of Human Resources, 35, 550-569.

Grima Camilleri, A. (2002). Comme c'est bizarre! L'utilisation d'anecdotes dans le développement de la compétence interculturelle. Strasbourg: Conseil de l'Europe.

Guo, S., \& Wong, L. (2015). Revisiting multiculturalism in Canada: Theories, policies and debates. Rotterdam, The Netherlands: SensePublishers.

Ham, S. H., Yang, K. E., \& Cha, Y. K. (2017). Immigrant integration policy for future generations? A cross-national multilevel analysis of immigrant-background adolescents' sense of belonging at school. International Journal of Intercultural Relations, 60, 40-50.

Joppke, C. (2018). War of words: interculturalism v. multiculturalism. Comparative migration studies, 6(11), 1-10.

Kahn, M. (2008). Multicultural education in the United States: Reflections. Intercultural Education 19(6), 527-536

Kymlicka, W. (2019). Deschooling multiculturalism. Ethnicities, 19(6), 971-982.

Leman, J. (1993). Les politiques d'éducation interculturelle dans la Communauté française et Communauté flamande de Belgique. Recherche et éducation, 15, 33-40 
LKenfack, J. (2017). Adolescents issus de l'immigration: mobilisation de ressources scolaires et communautaires en vue d'une meilleure insertion sociale. Ottawa, Canada: Université d'Ottawa.

Mathieu, F. (2018). The failure of state multiculturalism in the UK? An analysis of the UK's multicultural policy for 2000-2015. Ethnicities, 18(1), 43-69.

McLaren, P. (1997). Revolutionary multiculturalism: Pedagogies of dissent for the new millennium. Boulder, CO: Westview Press.

Meer, N., \& Modood, T. (2012). How does multiculturalism contrast with interculturalism? Journal of Intercultural Studies, 33(2), 175-196.

OCDE. (2015). Les élèves immigrés et l'école. Avancer sur le chemin de l'intégration. Paris, France: OCDE.

OECD. (2018a). Effective Teacher Policies: Insights from PISA. Paris, France: OECD.

OECD. (2018b). The resilience of students with an immigrant background: Factors that shape well-being. Paris, France: OECD.

Ogbu, J. U. (1987). Variability in minority school performance: a problem in search of an explanation. Anthropology and Education Quarterly, 18, 312-334.

Ogbu, J. U. (1991). Immigrant and involuntary minorities in comparative perspective. In A. M. Gibson \& J. U. Ogbu (Éds.), Minority Status and Schooling: A Comparative Study of Immigrant and Involuntary Minorities (pp. 3-33). New York, NY: Garland.

Perotti, A. (1994). Plaidoyer pour l'interculturel. Strasbourg, France: Conseil de l'Europe.

Porcher, L. (1981). L'éducation des enfants des travailleurs migrants en Europe: L'interculturalisme et la formation des enseignants. Strasbourg, France: Conseil de l'Europe.

Rangvid, B. S. (2007). Sources of immigrants' underachievement: Results from PISACopenhagen. Education Economics, 15(3), 293-326.

Schachner, M. K., He, J., Heizmann, B., \& Van de Vijver, F. J. (2017). Acculturation and school adjustment of immigrant youth in six European countries: Findings from the Programme for International Student Assessment (PISA). Frontiers in psychology, 8, 649.

Schnepf, S. (2007). Immigrants' educational disadvantage: an examination across ten countries and three surveys. Journal of population economics, 20(3), 527545.

Segura, E. (2011). L'interculturalisme québécois, alternative au multiculturalisme canadien? Voie québécoise ou voix québécoise. Humanisme et entreprise, 5, 81-88.

Sleeter, C. E. (2018). Multicultural Education past, present, and future: Struggles for dialog and power-sharing. International Journal of Multicultural Education, 20(1), 5-20.

Stepanovich, B. I. (2017). Main areas of the implementation of multiculturalism in professional educator training in the USA. Journal of Research in Educational Sciences (JRES), 8(10), 14-21.

Takaki, R. (2012). A different mirror: A history of multicultural America. Boston, MA: Little, Brown.

Taylor, C. (2012). Interculturalism or multiculturalism? Philosophy \& social criticism, 38(4-5), 413-423.

Thijs, J., \& Fleischmann, F. (2015). Student-teacher relationships and achievement goal orientations: Examining student perceptions in an ethnically diverse sample. Learning and Individual Differences, 42, 53-63.

Volante, L. (2018). The PISA Effect on Global Educational Governance. The PISA Effect on Global Educational Governance. New York, NY: Routledge.

Mots-clés: Interculturel, multiculturel, intégration, performances scolaires, PISA 


\section{Nationale Modelle für die Integration von Migrantinnen und Migranten: Auswirkungen auf die PISA-Ergebnisse?}

\section{Zusammenfassung}

Es gibt eine uralte Debatte zwischen denen, die glauben, dass interkulturelle Bildung der multikulturellen Bildung konzeptionell überlegen ist, und denen, die glauben, dass es keine signifikanten Unterschiede zwischen diesen beiden Modellen des Cultural Diversity Management gibt. In diesem Artikel analysieren wir zunächst die in dieser Debatte verwendeten Argumente. In einem zweiten Schritt werden wir die Ergebnisse der PISA-Umfrage nutzen, um die Ergebnisse von Ländern, die den Interkulturalismus übernommen haben (Frankreich, Spanien, Schweiz), mit Ländern zu vergleichen, die den Multikulturalismus befürworten (Kanada, USA, Großbritannien). Der Vergleich konzentriert sich auf die Ergebnisse junger Menschen mit unterschiedlichem Hintergrund (Migranten der ersten und zweiten Generation). Dieser Vergleich untersucht die möglichen Zusammenhänge zwischen Integrationsmodellen (Interkulturalität oder Multikulturalismus) und Schulleistungen, um zu klären, ob sich ein Modell tatsächlich vorteilhafter im Hinblick auf diese Fragen ausweist.

Schlagworte: Interkulturell, multikulturell, Integration, Schulleistungen, PISA

\section{Modelli nazionali per l'integrazione dei migranti: quale impatto sui risultati di PISA?}

\section{Riassunto}

Vi è un dibattito di lunga data tra coloro che ritengono che l'educazione interculturale sia concettualmente superiore all'educazione multiculturale e coloro che ritengono che non ci siano differenze significative tra i due modelli di gestione della diversità culturale. Nel presente articolo, vengono dapprima analizzati gli argomenti utilizzati nel dibattito. In una secondo momento, si utilizzano i dati dell'indagine PISA per confrontare i risultati dei paesi che hanno adottato l'interculturalità (Francia, Spagna, Svizzera) con i paesi che utilizzano il multiculturalismo (Canada, Stati Uniti, Regno Unito). Il confronto si concentra sui risultati dei giovani provenienti da contesti diversi (studenti immigrati di prima e seconda generazione). Tale confronto esplora i possibili collegamenti tra i modelli di integrazione (interculturalità o multiculturalismo) e le prestazioni scolastiche per vedere se un modello fa effettivamente la differenza su questi temi.

Parole chiave: Interculturalità, multiculturalismo, integrazione, rendimento scolastico, PISA 


\section{National models for the integration of migrants: what impact on PISA results?}

\section{Summary}

There is an age-old debate between those who believe that intercultural education is conceptually superior to multicultural education and those who believe that there are no significant differences between these two models of cultural diversity management. In this paper, we first analyse the arguments used in this debate. In a second step, we will use data from the PISA survey to compare the results of countries that have adopted interculturalism (France, Spain, Switzerland) with those that adopted multiculturalism (Canada, United States, United Kingdom). The comparison will focus on the outcomes of students from diverse backgrounds (first and second generation immigrant students). This comparison will explore the possible links between integration models (interculturalism or multiculturalism) and school performance to see if a model actually makes a difference on these issues.

Keywords: Intercultural, multicultural, integration, school performance, PISA

\section{Auteurs}

Thibaut Lauwerier. Titulaire d'une licence en sciences politiques, d'un master en études de développement et d'un doctorat en sciences de l'éducation, Thibaut Lauwerier est actuellement maître-assistant et collaborateur scientifique dans l'Équipe de de Recherche en Dimensions Internationales de l'Éducation (ERDIE). Ses recherches portent principalement sur l'analyse comparative et internationale de l'éducation.

Université de Genève, FPSE, Sciences de l'éducation, CH-1211 Genève

E-mail: thibaut.lauwerier@unige.ch

Abdeljalil Akkari. Après des études en sciences sociales à Lyon et en sciences de l'éducation à Genève, il a travaillé à l'université de Fribourg, à l'université de Baltimore et à la Haute École Pédagogique BEJUNE. Il est actuellement professeur en dimensions internationales de l'éducation à la FPSE à Genève et responsable du groupe de recherche ERDIE. Ses principaux travaux de recherche concernent l'éducation interculturelle, l'internationalisation des politiques éducatives et la coopération internationale en éducation.

Université de Genève, FPSE, Sciences de l'éducation, CH-1211 Genève

E-mail: abdeljalil.akkari@unige.ch 